\title{
La croissance urbaine en Roumanie
}

Violette Rey

\section{Citer ce document / Cite this document :}

Rey Violette. La croissance urbaine en Roumanie . In: Annales de Géographie, t. 91, n508, 1982. pp. 679-702;

doi : https://doi.org/10.3406/geo.1982.20156

https://www.persee.fr/doc/geo_0003-4010_1982_num_91_508_20156

Fichier pdf généré le 24/02/2020 


\begin{abstract}
What are the demographic, economic and spatial trends of Romanian urbanization ? Citydwellers are now as much as 10.3 millions ( $48 \%$ of total population) and city population has nearly doubled. The author describes the characteristics of this urbanization according to the State-ruled socialist ideological models and also to the more general usual urban-growth models.

First fed by the large rural population and the important natural growth, city growth is the result of migrations whose mode and size show gravity-like pattems. However this kind of urbanization seems to be phasing out right now, while temporary migrations and suburbanization develop altogether. Besides, the tertiary sector seems to outnumber the industry sector, which raises the crucial problem of the driving role of the tertiary sector within a socialist economy.

Spatial differences between the urbanization levels have attenuated but the origin of city neweomers and the consequences of rural exodus on the countryside notably differ on each side of a NW-SE diagonal. Five maps of the new conurbations that have developed since the beginning of this century show the densification steps of the national urban network (regular network, specific network). The voluntary spatial transformation of the population densities seems, according to this study, easier than the reorientation of city-size order, since the two rank-size graphs of 1930 and 1977 are alike.
\end{abstract}

\title{
Résumé
}

À quelles tendances démographiques, économiques et spatiales se rattache l'urbanisation roumaine? Elle atteint 10,3 millions de citadins (48\% de la population totale) et a presque doublé l'effectif des villes. L'auteur inscrit les modalités de cette urbanisation dans le cadre idéologique du socialisme d'Etat et dans les cadres plus généraux relatifs aux modèles de croissance urbaine.

La forte ruralité et la vitalité démographique ont d'abord nourri, de manière spécifique, la croissance, laquelle s'est appuyée sur des mouvements migratoires dont la forme et l'ampleur restent dans la dépendance des modèles de gravité. Toutefois, ce mode d'urbanisation paraît être en fin de phase, tandis que se développent une mobilité temporaire et la suburbanistion. Parallèlement, le secteur tertiaire semble prendre le relais sur le secteur secondaire, ce qui soulève l'importante question de la nature motrice du tertiaire socialiste.

Territorialement, il y a eu une réduction des écarts entre les niveaux d'urbanisation, mais avec un mode de recrutement urbain et des conséquences sur les campagnes profondément différents de part et d'autre d'une diagonale N.O.-S.E. Cinq cartes des nouvelles unités urbaines apparues depuis le début du siècle montrent les phases de densification du réseau national (réseau régulier, réseau spécifique). La transformation spatiale volontaire du semis de peuplement paraît, à travers cette étude, plus aisée que l'infléchissement de la hiérarchie des tailles, puisque les deux graphiques «rang-taille» de 1930 et 1977 sont similaires. 


\section{La croissance urbaine en Roumanie}

Violette Rey, Maître-Assistante Université de Paris I

Deux ensembles de caractères généraux doivent être rappelés dans la perspective de la croissance urbaine, l'un concerne l'Etat de la Roumanie, l'autre le système socialiste' ${ }^{\prime}$. La Roumanie est encore un pays fortement marqué par le fait rural; en 1948, elle n'avait que 3,7 millions de citadins, soit à peine le quart de sa population totale et les actifs agricoles représentaient $76 \%$ des actifs totaux; aucune ville ne dépassait 150000 habitants, hormis la capitale Bucarest qui jouissait par contre d'une position extrêmement dominante parce que déjà ville millionnaire. L'option socialiste, synonyme au plan socio-économique de la voie industrielle et de la généralisation du statut de salarié, a dû se réaliser sans les atouts objectifs que donnent un passé et une tradition industrielle (peu de main-d'œuvre qualifiée, quasi-absence de régions industrielles complexes...), ou que donne un territoire riche en ressources naturelles (malgré la présence de divers gisements dont en hydrocarbures). Ce pays, déjà du Sud de l'Europe orientale, est donc devenu un pays socialiste en développement, et l'objectif de ses dirigeants pour les années 1990 sera d'atteindre le stade de "pays à développement économique moyen". Dans ce contexte, la volonté d'urbanisation ne saurait se confondre avec la seule recherche de l'industrialisation, même si, dans la pratique, les deux phénomènes sont nécessairement corrélés. En effet, fidèle en cela au modèle socialiste soviétique, l'urbanisation est conçue comme le processus contemporain le plus global en matière de restructuration sociale,

1. Cet article développe une communication présentée à la Table Ronde "Croissance et structures urbaines dans les pays socialistes d'Europe centre orientale". Montpellier, université Paul Valéry, 5, 6, 7 février 1981 . 
comme la composante majeure de l'évolution de la formation sociale socialiste. De ce fait le concept d'urbanisation tel que l'emploient les autorités roumaines recouvre une signification proche de celle définie par E. Juillard à propos de l'urbanisation des campagnes ${ }^{2}$. Par ailleurs, c'est un objectif d'équilibre géographique d'égalisation des situations départementales qui guide la politique de "systématisation territoriale". Précisé au cours de la décennie soixante avec la nouvelle organisation administrative départementale (1967), cet objectif est depuis un leitmotiv des textes de planification territoriale, ce qui se traduit doublement au plan de l'urbanisation par la volonté de limiter la croissance des grandes villes au profit des villes moyennes et par le souci de constituer une trame urbaine très régulièrement maillée.

Résultat effectif de ces trente années d'évolution, la population urbaine roumaine a atteint les 10,3 millions d'habitants, soit $48 \%$ de la population totale, les actifs agricoles représentent $33 \%$ des actifs, le nombre des villes est passé de 152 à 236 avec comme projet d'atteindre les 505 unités en 1985. Ce large mouvement de croissance urbaine, je l'aborderai selon trois angles: celui de ses composantes; celui de sa relation avec les activités urbaines dominantes et motrices; celui des équilibres interurbains. Je préciserai au préalable la manière dont la ville roumaine est définie dans les statistiques.

\section{La ville roumaine relève d'une décision législative}

Sans reprendre ici l'ample question de ce qu'est la ville, de ce qui mérite le nom de ville, je montrerai à travers les définitions adoptées par la Direction Centrale de la Statistique quelle finalité est attribuée à cette entité spatiale. Les localités roumaines et les aires territoriales qui leur sont administrativement attenantes sont actuellement classées en trois types, les communes urbaines (villes et municipes), les communes suburbaines, les communes rurales. Chaque type est défini en fonction d'un complexe de critères, dans lequel l'usuel seuil d'effectifs d'habitants ne joue qu'un rôle limité. "La ville» reçoit son statut juridique de ville par décision législative.

«Elle doit être un centre fortement peuplé doté de fonctions politiques et administratives, d'un potentiel industriel commercial et socio-culturel significatif, et avoir de multiples liens avec sa région environnante sur laquelle elle exerce une influence économique et organisatrice. " 3

2. Faire que "les conditions de vie de la population rurale ressemblent toujours plus à celle des villes".

3. Plenum du C.C. du P.C.R. du 5-6 octobre 1967, statuant de l'amélioration de l'organisation administrative du territoire et de la systématisation des localités rurales. 
A ce statut s'attache donc un certain niveau d'équipement et d'autonomie de fonctionnement dans les deux domaines de la production et des services. "La commune suburbaine" est une commune de la proche périphérie d'une ville, qui a des liens de travail, transports et services avec le centre mais ne possède pas d'équipements socio-économiques autonomes. "La commune rurale» est en pratique définie par différence avec les précédentes. Une telle définition de la ville met en relief la conception retenue pour l'unité agglomérée comme un centre d'organisation sociale et de contrôle territorial. La mesure du processus de croissance urbaine sera donc étroitement liée à cette vision volontariste du législateur, ce qui n'est pas sans introduire certaines difficultés puisque ne sont pas précisés par des chiffres les seuils utilisés pour chacun des critères démographiques, morphologiques et fonctionnels retenus. Mais cette imprécision même témoigne de la place exceptionnelle accordée à la ville, outil, agent de la transformation globale de la société en direction d'une complexité croissante des activités et des relations. La liberté laissée au législateur pour attribuer le statut de ville n'a pas pour autant transformé toutes les bourgades en villes, lesquelles doivent avoir ou recevoir les équipements correspondants. Par ailleurs, je rappellerai que tout contenu urbain reste relatif à une forme de développement et de structure socio-culturelle, et que dans le cas présent l'image de la ville roumaine n'exclut ni les basses densités d'habitat, ni une activité agricole parfois très importante. Ainsi, la densité urbaine moyenne était de 47 habitants à l'ha en 1974, et, en 1976, 24 villes étaient classées agricoles parce que leur population active agricole était comprise entre 36 et $80 \%$ du total des actifs.

Les recensements exhaustifs de la population, principale source de documentation sur la croissance urbaine, ont eu lieu en 1930, $1948,1956,1966,1977$. Inévitablement tous ne relèvent pas des mêmes découpages territoriaux. En 1930 et 1948, la population urbaine départementale résidente comprend celle des villes et de quelques communes périphériques. De 1956 à 1965, elle comprend celle des villes et des 183 localités assimilées aux villes selon la décision $n^{\circ} 261$ du Conseil des ministres. En 1966, une nouvelle décision a augmenté le nombre des localités assimilées à 238. Le regroupement statistique dit de "l'unité urbaine" n'existe pas; la population de chaque commune urbaine est indiquée dans les annuaires statistiques annuels. Enfin il s'agit de la population résidente ayant son bulletin de domicile inscrit à la ville; ce qui exclut la population "flottante" qui habite plus ou moins durablement dans la ville où elle a un emploi, sans en avoir le bulletin de résidence. 


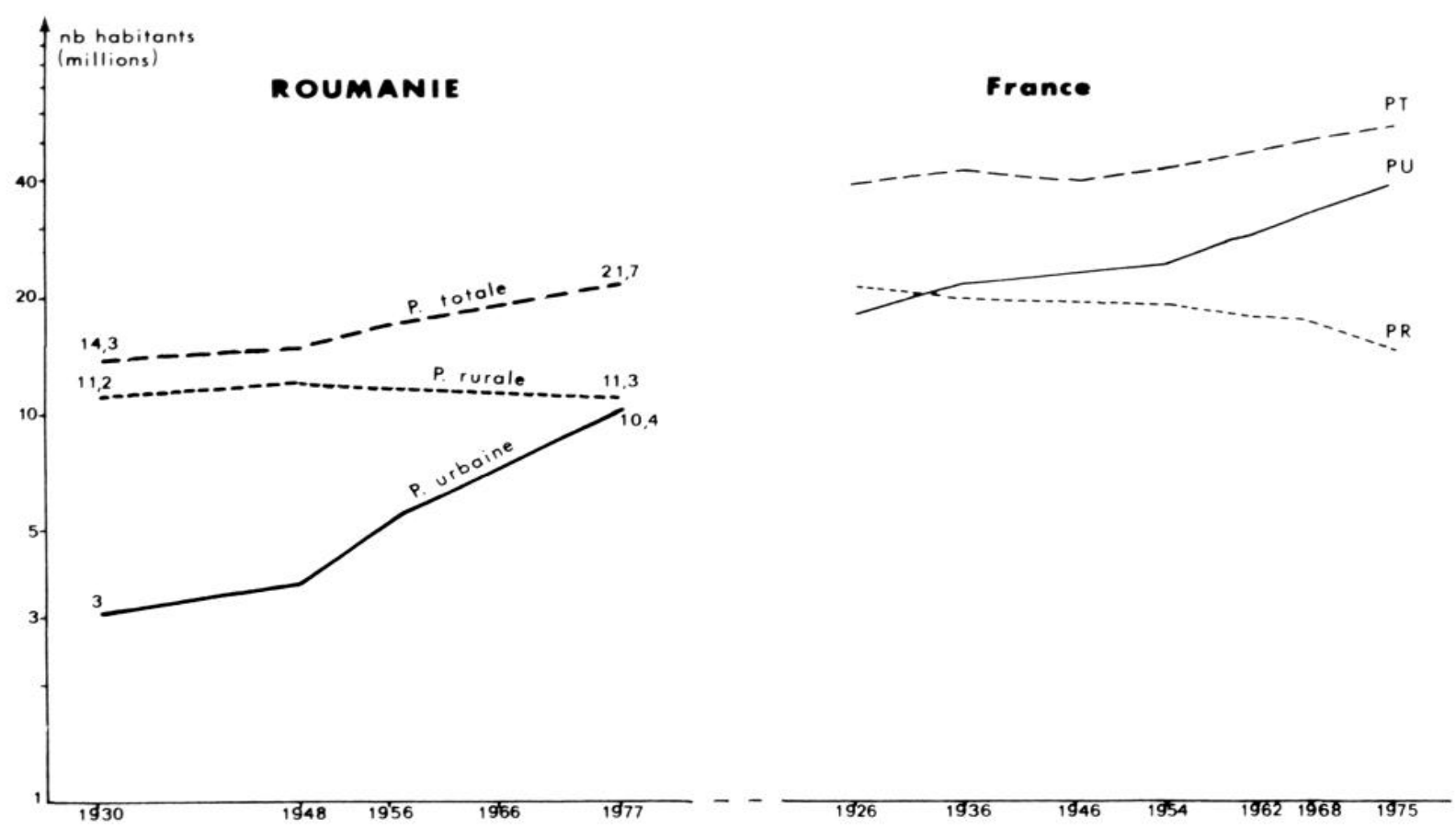

Fig. 1. - eVolutions démographiQues: populations urbaine, RuRAle, totale. RAPPEL DU MODÉLE DE CROISSANCE URBAINE D'APRÉS GIBBS (1961).

\footnotetext{
$1^{\text {er }}$ stade: les villes apparaissent, mais le taux de croissance de la Population rurale (PR) $\geqslant$ taux de croissance de la Population urbaine (PU)

$2^{\theta}$ stade: le taux de croissance PU devient $>$ taux de croissance PR

$3^{e}$ stade: le volume de la PR diminue

$4^{\circ}$ stade: la PU des petites villes tend à diminuer

$5^{\mathrm{e}}$ stade: les différences de densité de peuplement s'atténuent: il y a diffusion du phénomène urbain sur l'ensemble du territoire.
}

\section{La croissance urbaine et l'origine des citadins}

Du rythme général observé en Roumanie, je soulignerai l'intensité continue, mais particulièrement forte dans l'immédiate aprèsgucrre, lors de cette phase de profond bouleversement social et de déracinements multiples durant laquelle sont mis en place les premiers jalons de l'industrialisation socialiste. Il est notable de constater que la poursuite déterminée de la politique d'urbanisation se fait à une cadence actuelle plus modérée. Dans ce mouvement, c'est encore la croissance démographique globale qui est restée le principal moteur de l'urbanisation (fig. 1). Même si le point d'inflexion de la courbe de la population rurale date de 1948, la population rurale de 1977 est encore supérieure à celle de 1930; le transfert réel de la campagne vers la ville n'a jusqu'alors touché que les excédents ruraux, sans entamer quantitativement au moins l'effectif lui-même. Cette situation, liée à un fort dynamisme démographique roumain, est à souligner car elle place la Roumanie 
dans une position sans doute originale au sein des pays socialistes. La référence aux courbes françaises qui nous sont familières n'est là que pour mettre en valeur la différence du mécanisme: bien que cette période soit, en France, celle de la plus forte urbanisation, la pente de la croissance française est moins élevée que celle de la Roumanie et une grande part de cette croissance est alimentée par le déclin rural.

En d'autres termes, si l'on rapproche ces courbes du modèle dynamique proposé par $\mathrm{Gibbs}^{4}$ pour les pays développés, l'urbanisation actuelle de la Roumanie est à la charnière des phases II et III, lorsqu'on passe de la phase où le taux de croissance de la population urbaine est supérieur à celui de la population rurale, à la phase où le volume de la population rurale diminue. Cette situation charnière se traduit nettement au plan du comportement démographique des populations urbaines et rurales. Le décalage des taux de natalité et d'accroissement naturel qui jouait fortement en faveur des campagnes en 1956, s'est depuis réduit. En 1977, la natalité, encore élevée en milieu rural $(20,1 \% \circ$ contre $17,9 \%$ ), est en milieu urbain contrebalancée par le vieillissement relatif; désormais c'est en ville que le taux d'accroissement naturel est le plus élevé, (10,1\%o contre $8,6 \%$ à la campagne) parce que l'effet de moindre fertilité est compensé par un meilleur taux de nuptialité.

Expression d'un délestage des surplus démographiques, la croissance urbaine est nourric concrètement par d'amples mouvements migratoires, dont les tendances générales en matière d'origine des migrants et de distance de migration sont les suivantes. Les campagnes sont les principales pourvoyeuses de nouveaux citadins. Dans chaque bassin de migration, la quantité de nouveaux citadins fournie est inversement proportionnelle à la distance à la ville; elle se distribue selon une courbe très dissymétrique (de type exponentielle négative) telle que l'essentiel de l'apport provient de la zone proche (moins de $70 \mathrm{~km}$ ); au-delà de $150 \mathrm{~km}$ l'effet distance joue peu sur l'attraction, laquelle s'alimente au bassin national de recrutement urbain. L'attraction est en outre d'autant plus lointaine que la taille de la ville est plus grande (Measnicov et al., 1977). Ces deux caractéristiques montrent que l'urbanisation roumaine relève dans ses modalités spatiales du modèle de gravité si généralement observé ailleurs pour ce phénomène.

Comparativement à la période intercensale 1956-1966, la période 1966-1977 semble enregistrer deux modifications dans la forme de l'approvisionnement urbain. D'une part la poursuite de la croissance urbaine aux dépens de campagnes arrivées à des stades démographiques différents entraîne dans certaines l'apparition non

4. Gibbs, J.P., Handbook of urban research methods, Van Nostrand, 1961. 
seulement d'un vieillissement mais d'un dépeuplement absolu; dès situations démographiques régionales totalement nouvelles pour le pays, sont ainsi créées, que nous préciserons dans l'étude des équilibres territoriaux. D'autre part, il y aurait une certaine diminution des mouvements migratoires définitifs à grande distance mais la catégorie "population flottante", celle qui travaille trop loin de son domicile pour y résider, semble prendre une ampleur croissante, tout au moins, si l'on en juge par l'attention nouvelle que lui portent les travaux récents (Tufescu, 1978, Stefanescu et al. 1980). Enfin le mécanisme de subordination se développe doublement; par la progression de la population définie comme suburbaine (200 000 en 1966, 847000 en 1977); plus encore par l'augmentation du nombre d'actifs qui chaque jour quittent leurs communes rurales pour travailler en ville. Les multiples études cartographiques sur les aires de migrations quotidiennes et sur les transports ainsi mobilisés témoignent de la généralisation de ce phénomène (cf. Ianos et $M$. Olaru, 1980, et leur analyse sur l'optimisation des dessertes locales dans le département du Caras Severin).

\section{La croissance urbaine et les activités dominantes}

Sans doute est-ce au niveau de la relation entre la croissance urbaine et la nature des activités urbaines que la spécificité du modèle socialiste intervient apparemment le plus nettement et que les problèmes d'analyse et de comparaison sont les plus délicats, compte tenu des différences de conceptions sur la nature et l'organisation du développement économique et sur la terminologie utilisée pour le décrire. La promotion de la ville par l'industrie, la création des villes nouvelles sur des foyers industriels ont été les réalisations les plus spectaculaires en matière d'urbanisation socialiste et proposées souvent comme l'expression urbaine spécifique de ce système socio-politique de développement. Cependant, l'évolution technologique aidant, la "révolution tertiaire" va-t-elle ici aussi succéder à la "révolution industrielle "? Telle est la question que l'on peut poser dans le cas de la Roumanie. Des nombreuses typologies établies par les géographes roumains pour décrire la structure du système urbain de leur pays, en connaître la genèse, en évaluer les dynamismes potentiels, et pour en proposer les améliorations fonctionnelles conséquentes, je retiendrai les résultats suivants en relation avec la croissance urbaine.

Sur moyenne période, la typologie proposée $^{5}$ distingue six

5. Atlas national de la République socialiste de Roumanie, Edition de l'Académie, planche IX. 
catégories de villes selon la forme de leur croissance: les villes à croissance "explosive », celles à croissance "ascendante équilibrée », celles à croissance "modérée »; puis, celles à évolution « modérée stagnante", celles "stagnantes ou transitoires», celles enfin à évolution "oscillante" et parfois "régressive". A travers cette typologie, il n'est pas possible d'établir de relation systématique entre la croissance et la taille des centres ou un secteur d'activité; la mono-activité, même si elle est de nature industrielle, se montre parfois préjudiciable à la croissance soutenue de nouveaux centres urbains.

En effet la relation entre industrialisation et croissance urbaine, si fortement mise en évidence par les études des années 1960, paraît appartenir à la première phase (1950-1965), lorsque l'industrialisation a touché toutes les villes et leur a donné des profils d'activités beaucoup plus semblables en 1956 qu'en 1930 (Sandru, 1961).

Cependant depuis une décennie, le secteur tertiaire semble prendre le relais sur le secteur secondaire comme moteur de la croissance $^{6}$. C'est ainsi que Measnicov et al. (1977) ont classé leur échantillon de 170 villes selon l'activité dominante en 1966: 23 étaient villes "primaires» (population active agricole de 37 à 80\%), 87 étaient villes "secondaires" (PA industrielle de 35 à 85\%), 60 étaient villes "tertiaires" (PA tertiaire de 37 à $72 \%$ ). Ils ont évalué la croissance démographique propre à chaque type, entre 1956-1966 et 1966-1974 et ils ont mis en relief qu'à la phase de croissance prééminente des centres industriels succède celle des centres tertiaires. Ce changement est confirmé par les résultats du recensement de 1977, et j'ajouterai que s'accentue l'écart de dynamisme entre les villes selon leur type d'activité (tabl.1). Enfin si l'on observe la

TABLEAU 1

\section{Croissance urbaine et activités dominantes}

Indice de croissance de la population active des villes, selon:

\begin{tabular}{c|c|c|c}
\hline le type de ville... & «primaire » & «secondaire » & «tertiaire » \\
\hline $1956-1966(1956=100) \ldots \ldots \ldots \ldots \ldots \ldots \ldots \ldots \ldots$. & 123 & 137 & 135 \\
$1966-1974(1)(1966=100) \ldots \ldots \ldots \ldots \ldots \ldots \ldots$. & 108 & 120 & 140 \\
$1966-1977(2)(1966=100) \ldots \ldots \ldots \ldots \ldots \ldots \ldots$ & 112 & 141 & 162 \\
\hline
\end{tabular}

(1) D'après Measnicov et al. (1977), sur l'échantillon des 170 centres considérés villes au RGP de 1956. (2) D’après Cucu et al. (1978), pour l'ensemble des villes. Cet article indique en outre que les villes chefs-lieux de département ont cu un taux global de croissance de $+267 \%$ entre 1948 et 1977 , contre seulement $+210 \%$ pour les autres.

6. Je rappelle que le secteur tertiaire est non sculement moins important à cause de l'organisation socialiste de la société mais en plus sous-évalué statistiquement puisque les emplois de services intégrés dans les entreprises industrielles ou agricoles sont rattachés aux secteurs primaire et secondaire. 
croissance en fonction de la place de la ville dans le réseau hićrarchique politico-administratif, il apparaît que les chefs-lieux des départements, les villes relais dans l'élaboration des décisions, dans la gestion du fonctionnement et dans le contrôle de l'organisation globale, c'est-à-dire des villes par définition dotées d'attributs tertiaires, ont une croissance nettement plus forte que les autres (Cucu, 1978).

Quel poids accorder à ce phénomène? Est-il l'amorce du cycle de tertiarisation qui succéderait à celui de l'industrialisation comme le processus en a été constaté en maint autre pays (Schultz, 1978 ${ }^{7}$ ). Est-il l'expression d'une nouvelle configuration de l'identité urbaine et de la nature de la ville? Ce qui serait d'un intérêt théorique évident puisque cela obligerait à reprendre la réflexion sur le contenu et le rôle du tertiaire dans le système urbain socialiste, lequel avait été dévalorisé dans l'optique productiviste. En fait, l'avantage que prennent ici les fonctions tertiaires dans la croissance urbaine roumaine, ne correspond pas (ou tout au moins pas encore) à une tertiarisation nettement croissante de l'économie roumaine. Il est vrai que le recul du secteur agricole laisse une plus large place au secteur tertiaire $(14 \%$ de la population active en 1966, 24\% en 1977); néanmoins c'est le taux de croissance du secteur industriel qui reste le plus fort (tabl.2). La tendance nouvelle de la croissance urbaine renvoie donc à des mécanismes sélectifs de la croissance actuelle entre villes, et nécessitera des études plus fines sur les croissances spécifiques en liaison avec les activités, décomposées selon une nomenclature détaillée. Travail long et complexe, mais prometteur pour la connaissance de la

TABLEAU 2

Indices d'accroissement de la population active par grands secteurs d'activité (en milliers d'actifs)

\begin{tabular}{l|r|r|r|r|c|c|c}
\hline & 1950 & 1960 & 1970 & 1977 & $1960 / 1950$ & $1970 / 1960$ & $1977 / 1970$ \\
\hline Total............. & 8377 & 9538 & 9875 & 10264 & 114 & 103 & 104 \\
& $100 \%$ & $100 \%$ & $100 \%$ & $100 \%$ & & & \\
Industrie et & 1187 & 1910 & 3044 & 4364 & 161 & 159 & 143 \\
construction ..... & $15 \%$ & $21 \%$ & $31 \%$ & $41 \%$ & & & \\
Agriculture, & 6226 & 6250 & 4867 & 3374 & 100 & 78 & 69 \\
sylviculture....... & $74 \%$ & $65 \%$ & $49 \%$ & $35 \%$ & & & \\
Autres............ & 964 & 1377 & 1964 & 2549 & 143 & 151 & 130 \\
& $11 \%$ & $14 \%$ & $20 \%$ & $24 \%$ & & & \\
\hline
\end{tabular}

Anuarul statistic R.S.R., 1979, Bucarest.

7. Schulte (1978), "Les villes de Hongrie, le róle des villes dans la société socialiste", Bulletin de la Société languedocienne de géographie, $285 \mathrm{p}$. 
dynamique du système urbain roumain et qui permettrait de dépasser la dichotomie trop formelle entre secteurs secondaire et tertiaire. Ce travail gagnerait en outre à s'articuler sur les comportements des villes face aux flux migratoires. Rien à ma connaissance n'a été récemment écrit sur le degré de capacité des villes à fixer leur apport migratoire, même si des indications en ce sens sont fournies dans les études régionales et montrent le taux élevé de rotation des travailleurs dans les centres industriels nouveaux.

\section{La croissance urbaine et les équilibres interurbains}

La problématique d'aménagement territorial des dirigeants roumains, comme je l'ai rappelée en introduction, a pour principe d'assurer de meilleurs équilibres territoriaux, entre les villes et les campagnes, entre les villes entre elles. Sans discuter ici de ce que recouvre un tel principe d'égalisation, j'ai donc cherché à évaluer dans la diffusion géographique de la croissance urbaine roumaine le degré d'homogénéisation territoriale acquis. Cette opération retrouve d'ailleurs toutes les difficultés liées à l'évolution de la croissance urbaine elle-même. J'ajouterai que la constation d'une accentuation ou d'une réduction des contrastes dans la répartition de certaines composantes de la croissance ne permet pas seule de porter un jugement sur la tendance vers l'équilibre. Pour porter un tel jugement il faudrait disposer de beaucoup d'autres éléments que nous n'avons pas. Toutefois, j'envisagerai la dimension géographique de la croissance urbaine et sa contribution à l'équilibre territorial à partir des critères les plus simples des niveaux départementaux d'urbanisation et des trames urbaines.

C'est bien sur l'ensemble du territoire que l'urbanisation progresse et l'évolution des taux départementaux d'urbanisation montre une réduction progressive des écarts entre les départements les plus et les moins urbanisés (tabl.3). L'amplitude fut maximale en 1956, après la phase des fortes mutations sociales d'après guerre, et la réduction apparaît surtout sensible depuis 1966; en 1977, les taux oscillent entre $24 \%$ en Bistrita Nasaud et $72 \%$ dans le pays noir de Hunedoara. La carte de l'urbanisation (fig. 2) est un calque des zones de fort développement économique, avec principalement l'axe industriel central (du Timis au Prahova) et la zone du littoral et du Bas Danube. Par contre la variation du taux d'urbanisation entre 1948 et 1977 montre, au plan départemental, une répartition tranchée, opposant, parmi les départements dont l'urbanisation initiale était faible, ceux dont l'accroissement moyen a triplé et ceux qui n'ont pas encore atteint le doublement (fig. 3). Le premier 
TABLEAU 3

Evolution de l'urbanisation 1930-1977

A - Effectifs et taux d'urbanisation

\begin{tabular}{|c|c|c|c|c|c|}
\hline & 1930 & 1948 & 1956 & 1966 & 1977 \\
\hline Population totale.. & 14280 & 15872 & 17389 & 19103 & 22070 \\
\hline Population urbaine ..................... & 3051 & 3713 & 5474 & 7305 & 10362 \\
\hline Taux départemental de P.U. . ........... & $21 \%$ & $23 \%$ & $31 \%$ & $38 \%$ & $48 \%$ \\
\hline
\end{tabular}

B - Nombre de villes par classe de taille

\begin{tabular}{|c|c|c|c|c|c|c|}
\hline & 1930 & 1948 & 1956 & 1966 & 1977 & $1985^{*}$ \\
\hline 500000 (Bucarest)............ & $21 \%$ & $\begin{array}{c}1 \\
28 \%\end{array}$ & $\begin{array}{c}1 \\
25 \%\end{array}$ & $\begin{array}{c}1 \\
20 \%\end{array}$ & $\begin{array}{c}1 \\
19 \%\end{array}$ & \\
\hline $200-300000 \ldots \ldots$ & 0 & 0 & 0 & 0 & $\begin{array}{c}8 \\
23 \%\end{array}$ & \\
\hline $100-200000 \ldots \ldots$ & 3 & 2 & $\begin{array}{c}7 \\
18 \%\end{array}$ & $\begin{array}{c}12 \\
26 \%\end{array}$ & $\begin{array}{c}9 \\
13 \%\end{array}$ & 19 \\
\hline $50-100000 \ldots \ldots$ & 6 & 9 & $\stackrel{9}{14} \%$ & $\begin{array}{c}9 \\
8 \%\end{array}$ & $\begin{array}{c}18 \\
13 \%\end{array}$ & 77 \\
\hline $20-50000 \ldots \ldots \ldots \ldots$ & 15 & 14 & $\begin{array}{c}25 \\
16 \%\end{array}$ & $\begin{array}{c}43 \\
19 \%\end{array}$ & $\begin{array}{c}57 \\
17 \%\end{array}$ & \\
\hline $10-20000 \ldots \ldots \ldots$ & 37 & 32 & $\begin{array}{c}60 \\
18 \%\end{array}$ & $\begin{array}{c}76 \\
16 \%\end{array}$ & $\begin{array}{c}69 \\
10 \%\end{array}$ & 269 \\
\hline $10-5000 \ldots \ldots$ & 46 & 44 & 50 & 76 & 60 & \\
\hline $5000-0 \ldots \ldots \ldots \ldots \ldots$ & 35 & 51 & 20 & 19 & 14 & \\
\hline Total................ & $\begin{array}{c}142 \\
100 \%\end{array}$ & $\begin{array}{c}152 \\
100 \%\end{array}$ & $\begin{array}{c}171 \\
100 \%\end{array}$ & $\begin{array}{c}236 \\
100 \%\end{array}$ & $\begin{array}{c}236 \\
100 \%\end{array}$ & 365 \\
\hline
\end{tabular}

- Dans chaque classe, la $1^{\text {rc }}$ ligne représente le nombre de villes

la $2^{\mathrm{e}}$ ligne le $\%$ de la population urbaine de la classe de ville par rapport à la population urbaine totale à la date donnée.

* D'après J. Popovici et al. (1980), "Le développement économique et social de la Roumanie dans les années 1980, en perspective territoriale", R.R. 666, pp. 3-12.

groupe ceinture en une demi-couronne le foyer industriel central; l'autre correspond à la frange orientale du pays. En d'autres termes cette carte suggère que l'égalisation des taux d'urbanisation s'opère selon une assez nette contiguité spatiale en diffusant de part et d'autre des noyaux urbanisés en 1948 (Brasov, Sibiu, Cluj, Ploesti).

Effet d'intenses mouvements migratoires, l'urbanisation roumaine a d'abord été le fruit d'un comportement démographique à forte fécondité de la population roumaine. D'où l'importance que l'on doit accorder aux comportements comparés de la population urbaine et de la population rurale dans chaque département, à la fois pour apprécier les stades d'évolution auxquels sont parvenus les différentes populations régionales et pour cerner le devenir des renouvellements démographiques régionaux. Les quatre types de 

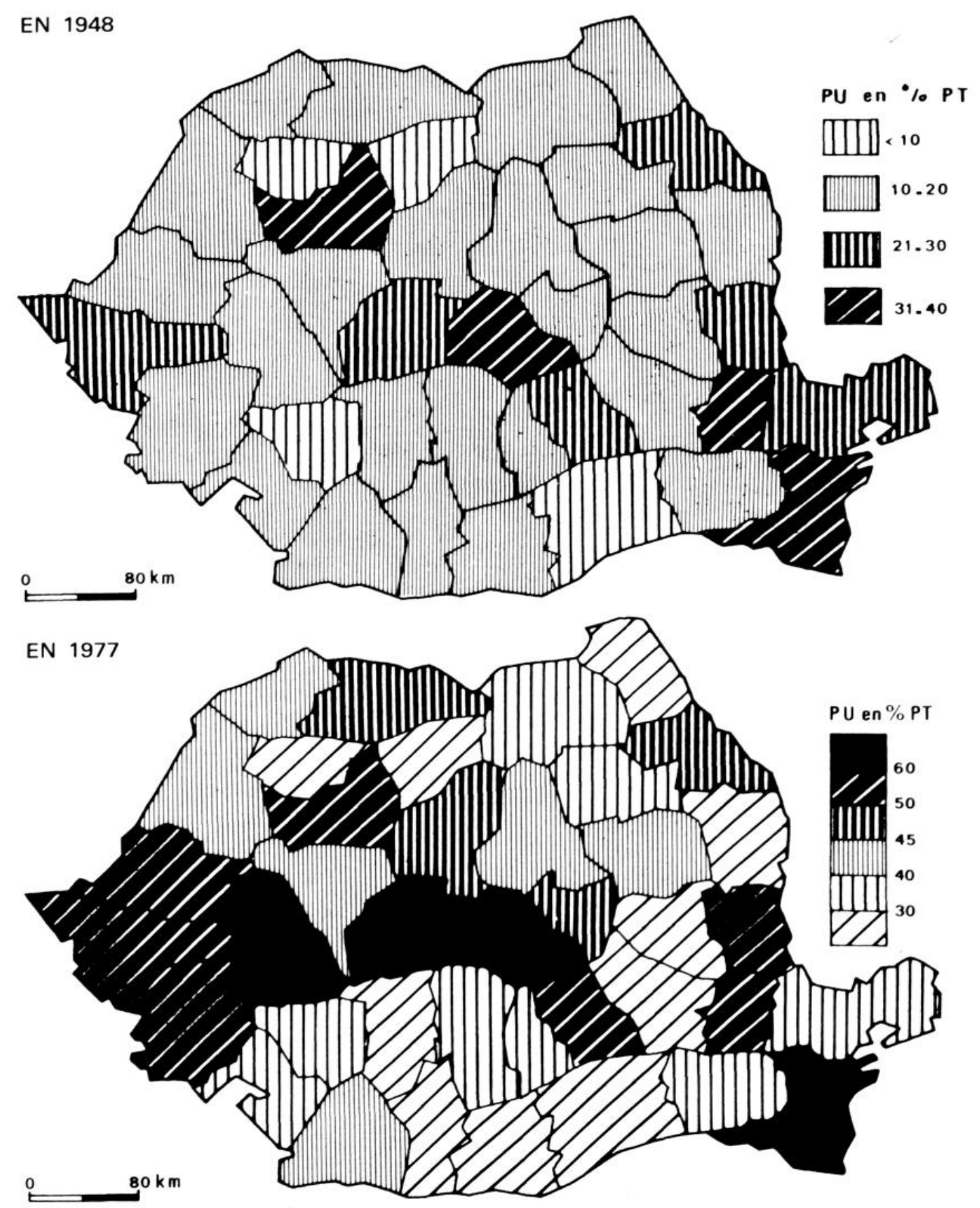

Fig. 2. - TAUX D'URBANISATION DES DÉPARTEMENTS ROUMAINS.

situations démographiques possibles existent effectivement (fig. 4). Un accroissement naturel nettement supérieur à l'accroissement départemental moyen aussi bien en zone urbaine que rurale est la caractéristique de la partic orientale du pays, dont j'ai précédemment souligné l'urbanisation modérée et en faible croissance; apparaît ici cette forte fécondité moldave qui permet encore aux villages moldaves d'accroître leurs effectifs. A l'opposé, dans le type 


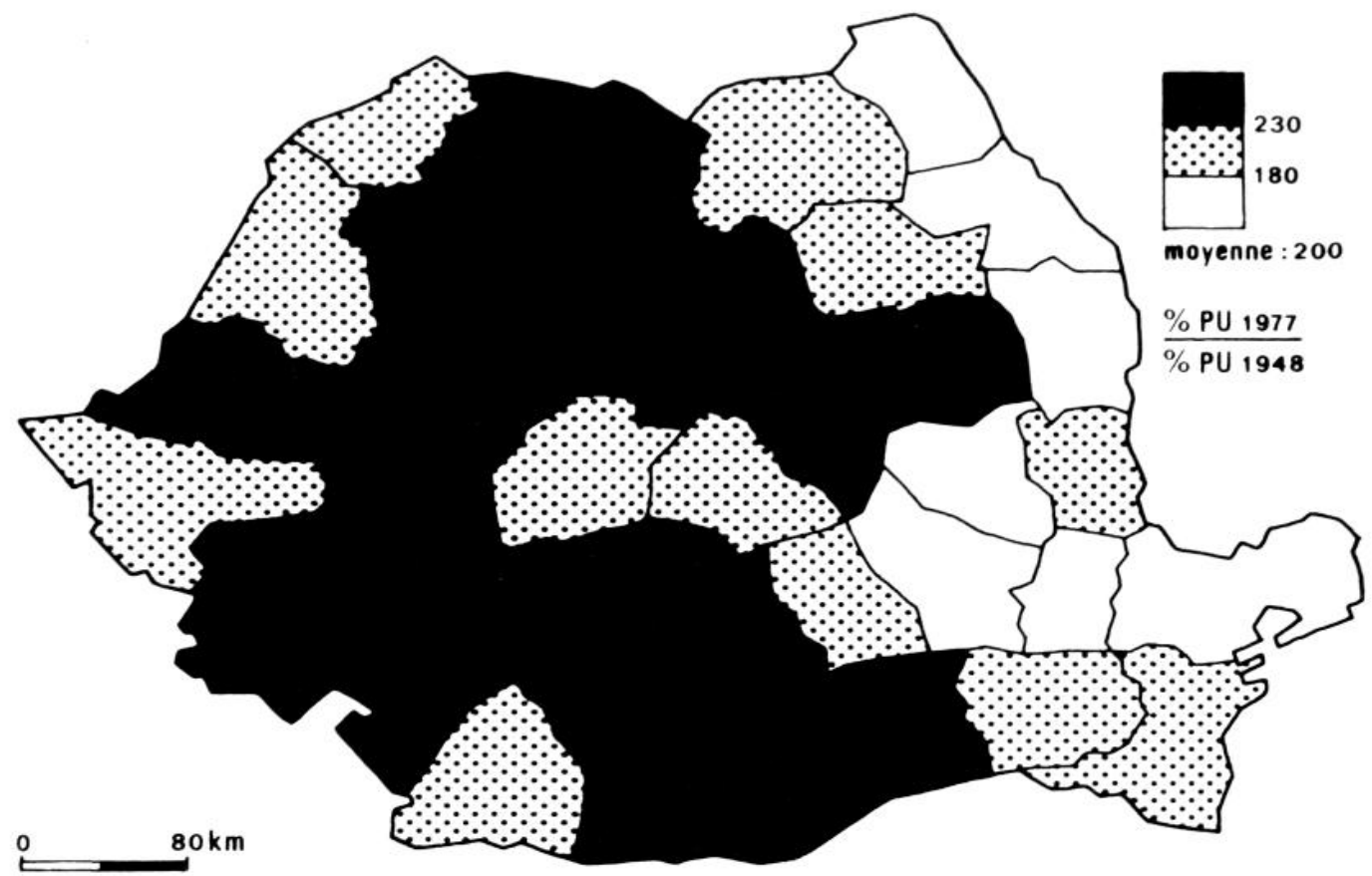

Fig. 3. - VARIATION DU TAUX D'URBANISATION 1948-1977.

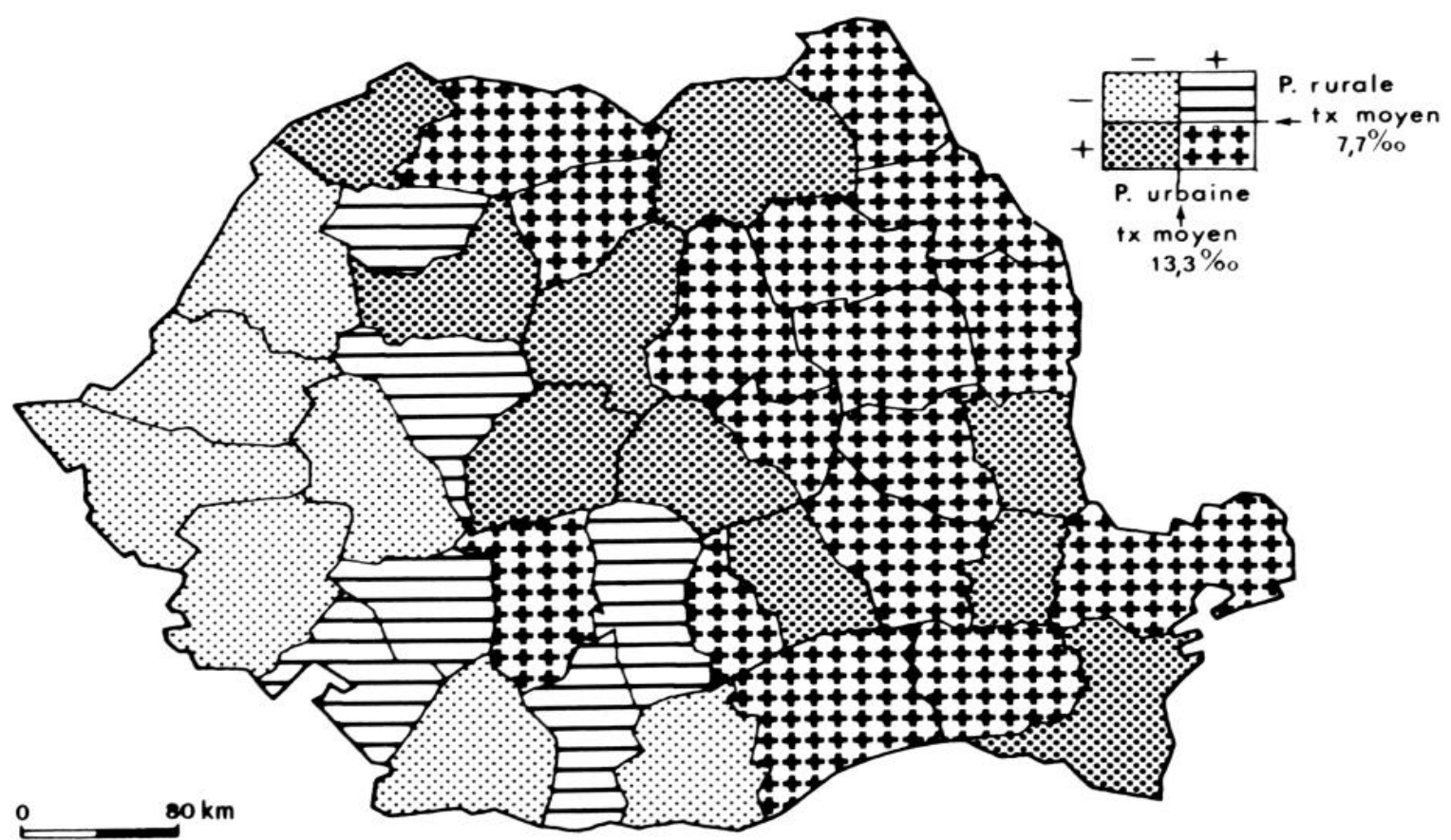

Fig. 4. - TYPES DÉPARTEMENTAUX DE COMPORTEMENT DÉMOGRAHIQUE DES POPULATIONS URBAINES ET RURALES (1977)

(TAUX D'ACCROISSEMENT NATUREL).

"banatique " occidental, les taux sont très faibles en zone urbaine (inférieurs à 5\%o) et même négatifs en zone rurale; ce type commence à empiéter sur la Basse Valachie (département du Dolj et de Telcorman). Un troisième type est caractérisé par l'opposition entre un accroissement naturel élevé en zone urbaine et très bas en 
zone rurale, affectant le piémont valaque et deux départements montagneux de la Transylvanie occidentale. Ce type correspond à une urbanisation très récente; le passage de la campagne à la petite ville n'a pas encore ralenti la fécondité du type rural, mais les campagnes, ici depuis longtemps pourvoyeuses de nouveaux citadins pour l'ensemble de la Roumanie, n'ont plus pour elles-mêmes qu'une population vieillie et à excédent naturel très limité. Le quatrième type juxtapose deux accroissements naturels modérés, légèrement inférieur au taux moyen urbain en zone urbaine, légèrement supérieur au taux moyen rural en zone rurale. Ce type correspond aux départements anciennement marqués par la présence d'une grande ville (Cluj, Sibiu, Brasov...), ayant atteint le développement économique le plus complexe et souvent le meilleur niveau de vie. En cela ce type semble correspondre à la forme de comportement démographique qu'atteignent les régions à urbanisation évoluée.

On constate donc que de fortes différences structurelles en matière de renouvellement démographique urbain et rural partagent le territoire roumain en deux, selon une diagonale approximativement N.O.-S.E.: à l'ouest de la ligne, existe un développement urbain marqué mais inscrit sur des campagnes déjà ou prochainement désertées; à l'est de la ligne, les campagnes dominent, avec un fort surplus démographique porteur d'une urbanisation potentielle. Ce partage est la résultante d'effets proprement économiques dûs au développement industriel combinés à des effets plus spécifiquement démographiques. En quoi, peut-on alors s'interroger, les migrations inter-régionales contribuent-elles à réduire ou accentuer ce partage territorial? De l'avis de Measnicov (1977, p. 95), et contrairement à l'hypothèse que l'on pourrait facilement avancer sur la pression démographique rurale et la surpopulation agricole comme facteurs de migration à la ville, la corrélation entre le taux d'accroissement naturel global et le taux de départ hors du département est nulle $(r=-0,17)$, au moins jusqu'en 1966. Les Transylvains du Nord et les paysans valaques ont plus tôt et en plus grand nombre quitté leurs villages que les Moldaves. Cette observation met en valeur des différences d'attitudes régionales face à la mobilité, elle traduit vraisemblablement aussi l'effet de cette loi entre la longueur du déplacement et le volume des départs, puisque la Moldavie s'est trouvée être la plus éloignée des zones à forte expansion industrielle et urbaine des années 1950-1960. Toutefois, les conclusions seront vraisemblablement modifiées quand on connaîtra les analyses portant sur la période 1966-1977; la zone centre occidentale ayant de moins en moins de surplus démographiques susceptibles d'alimenter la demande de ses grands 
centres industriels, son attraction migratoire portera jusqu'en Moldavie. D'ailleurs la carte de la population "flottante" en 1977 montre ce même partage du territoire en deux, au profit principalement de la zone centre-occidentale (fig. 5, d'après M. Apavaloaei, 1980).

La seconde façon d'approcher la dynamique de la répartition de l'urbanisation et d'apprécier l'effort consenti en matière d'équilibrage passe par l'étude de l'évolution des villes elles-mêmes, évolution mise en rapport avec le double objectif politique de l'Aménagement du Territoire, celui d'augmenter le nombre de villes plutôt que de laisser croître la taille des grandes villes, celui d'en assurer la meilleure équi-répartition spatiale.

En matière de nombre de villes et de hiérarchie de tailles entre elles, la croissance urbaine contemporaine a réussi à ne pas accentuer l'écrasante primauté de la capitale mais à la réduire légèrement et elle a assuré l'apparition d'un nombre important de nouvelles unités. Bucarest, avec 1060000 habitants en 1948 concentrait $28 \%$ de la population urbaine du pays; en 1977 ses 1934000 habitants n'en représentent plus que $19 \%$ (à la différence avec l'évolution de Budapest qui atteint $40 \%$ de la population urbaine hongroise). Quant au nombre de villes, il passe de 152 à 236 entre les deux dates, alors que stagnent ou même régressent les effectifs de villes dans les démocraties socialistes du Nord (Pologne, RDA...); ces mouvements sont à rattacher à l'état antérieur de chaque réseau national. En matière de croissance spécifique selon les tailles, il faudrait suivre les évolutions des villes en fonction de leurs tailles initiales (en 1930 ou en 1948) pour évaluer comment l'action d'incitation et de contrôle de la croissance a pu tenir compte de la taille moyenne souhaitée.

Le tableau 3 répartissant la population urbaine à chaque date selon la catégorie de taille des villes ne permet pas de porter un jugement sur les croissances spécifiques des catégories, même si transparaît un effet global de transfert au profit des catégories supérieures. Seul le graphique dit de Zipf, ordonnant les villes (fig. 6) selon leur rang et leur taille permet une appréciation des tendances spécifiques entre 1930 et 1977. Le tracé de 1977, parallèle à celui de 1930, montre qu'en Roumanie pas plus qu'ailleurs la modification de la hiérarchie n'a été très forte, malgré l'ampleur de la mutation politico-économique intervenue depuis $1945^{8}$. L'inertie de la forme d'une répartition rang-taille à l'intérieur d'un système urbain national est donc toujours très importante. La similitude des tracés aux deux dates, entre lesquelles s'inscrit pourtant un

8. Voir a ce sujet D. Pumain, 1980 "Contribution a l'etude de la croissance urbaine dans le svisteme urbain français" these d'Etat, Université de Paris I. 


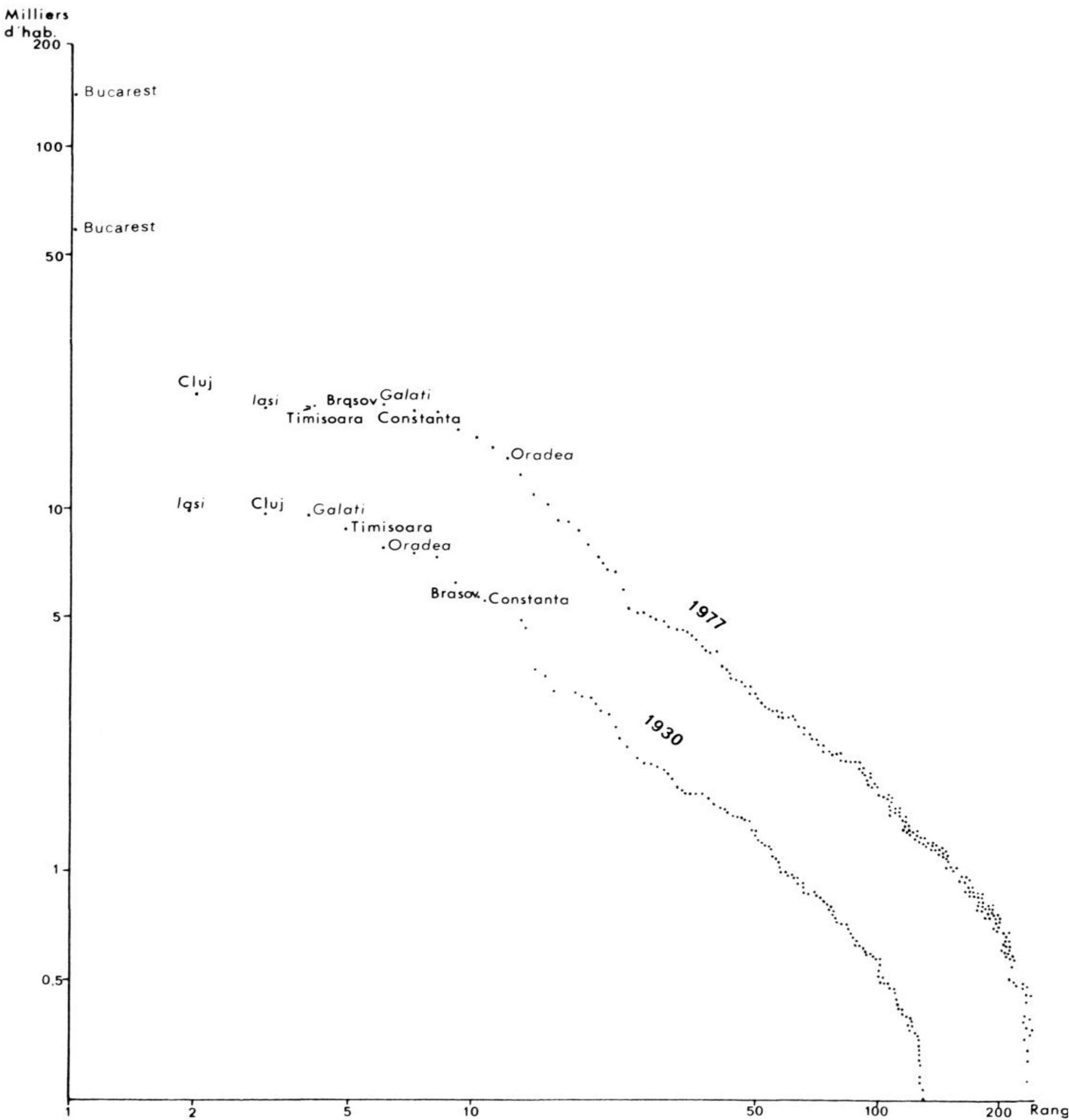

Fig. 6. - L'ORDRE DES VILLES SELON LEUR TAILLE, 1930, 1977

(d'après Panaite, Chitu, Caloianu, 1978). 
triplement de la population urbaine, signifie donc que la quantité de nouveaux citadins roumains a été proportionnelle à la taille initiale de chaque ville, et que les taux de croissance sont en moyenne identiques et indépendants des tailles. Ceci n'exclut pas certaines redistributions internes de rang, comme le montre le tracé des trajectoires (exemple de Brasov qui passe de $11^{\circ}$ au $4^{\mathrm{e}}$ rang), ni le renforcement relatif de la part des grandes villes, tandis qu'un certain nombre ont subi un recul dans la catégorie des villes moyennes. C'est donc plus du côté de la trame spatiale que de la forme de la hiérarchie que sont à rechercher les modifications spontanées ou volontaristes introduites dans la croissance urbaine.

La transformation spatiale volontaire du semis de peuplement est-elle paradoxalemet plus facile que l'infléchissement de la hiérarchie des tailles? Alors même qu'on touche là à l'implantation physique des villes donc à un élément incommensurablement plus fixe que les populations elles-mêmes. Là encore, l'évaluation d'une transformation de ce type ne peut-être correctement tentée que sur une durée suffisamment longue. J'ai donc cherché à insérer la volonté actuelle d'équilibre de répartition territoriale des villes dans l'histoire du réseau urbain roumain, en détachant les trames successives des nouveaux établissements urbains apparus depuis $1912^{\circ}$. De la cartographic de ces étapes on peut dégager les conclusions suivantes (fig. 7). En 1912 (à un moment où l'unité roumaine n'était pas encore achevée), la régularité de la distribution l'emporte, même si la ligne de contact des piémonts transparaît, même si la densité est moindre dans les basses plaines le long du Danube ou à la bordure de la plaine hongroise. Il s'agit d'un réseau urbain pré-industricl typique où les villes, lieux d'échanges avec leurs campagnes environnantes, sont assez uniformément espacées, compte tenu des déviations introduites par l'agencement du relief. Entre 1912-1930, 27 nouveaux centres apparaissent surtout à l'ouest de l'Olt et le long du Siret, et ils assurent une amélioration de la régularité de répartition du semis. La rupture de cette tendance remonte aux années 1930: 8 des 9 villes apparues entre 1930 et 1948 sont concentrées dans le triangle entre Sibiu, Ploesti, et Onesti. L'émergence du cœur économique du pays au niveau de la courbure carpatique, à cheval sur la Transylvanie du Sud et la Munténic s'individualise. La localisation de la quarantaine de créations effectuées entre 1948-1966 lors de la première phase d'industrialisation socialiste est précisément dictée par la présence des gisements à mettre en valeur: les zones montagneuses renforcent leur avantage; le semis des nouvelles unités a la forme bien

9. D'après l'Atlas national déjà cité: 1912, 1930, 1948, 1966, 1977: les trois premiers intervalles sont de 18 ans et justifient pleinement de la comparaison directe des cartes. 

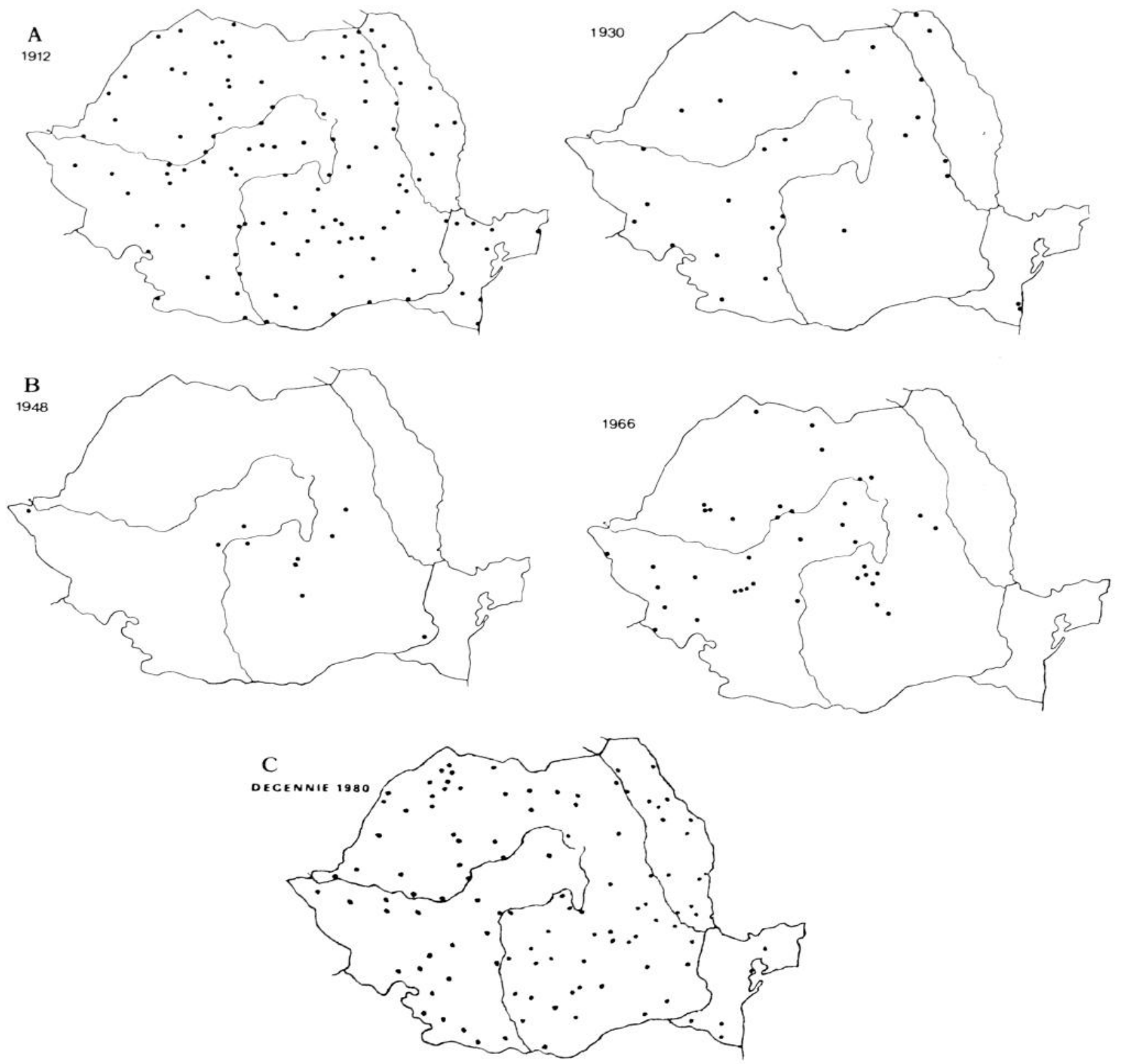

Fig. 7 - ÉVOLUTION DE LA TRAME URBAINE DE ROUMANIE: SITUATION EN 1912 UNITÉS URBAINES APPARUES JUSQU'EN 1930, 1948, 1966, 1980.

A. Trame urbaine pré-industrielle à semis régulier.

B. Urbanisation de type industriel à semis discontinu et concentré.

C. Urbanisation des campagnes avec régularisation du semis (localisation des bourgades qui seront prochainement déclarées villes).

particulière des urbanisations industrielles en grappe ou en chapelet, autour des gisements (Petrosani) ou le long de certaines vallées (Prahova). A partir de 1966, ce potentiel d'urbanisation spécifique est à peu près complètement mis en valeur (avec parfois même des régressions de petits centres miniers qui n'avaient d'urbain que le nom). Il y a retour à un semis plus banal des centres promus au 
rang de villes, choisis en fonction de leur coefficient local de centralité, aidés par la diffusion des ateliers d'industries légères, et dont la charte de systématisation du territoire en a fondé politiquement la finalité en 1967. Leur répartition reste cependant relativement spécifique puisqu'elle ne touche que les deux ensembles du N.O. (bassins du Somes et du Cris) et du Sud (Valachie), dont j'ai souligné la situation démographique en voie de vieillissementappauvrissement et la moindre densité de villes dès 1912. L'effort certain de diffusion du fait urbain a donc porté sur les régions centrales et occidentales, où la présence urbaine était plus forte mais le support démographique plus fragile, avant de s'implanter sur la partie orientale du pays pourtant soumise à une très nette pression démographique rurale. Qu'en sera-t-il de la trame du lot de créations de 1980-1985? Les 130 bourgades, dont on projette le passage au rang de ville dans cette décennie, sont réparties beaucoup plus uniformément, et témoignent de la volonté d'urbanisation des campagnes; d'ailleurs le projet stipule que chaque département devra avoir au moins 7 villes en 1985, alors même que les densités globales oscillent de 30 à 174 habitants $/ \mathrm{km}^{2}$.

La volonté d'équirépartition territoriale systématique se traduit peut-être plus encore dans la hiérarchie fonctionnelle des centres que dans le simple semis. Dès 1973, j'en soulignais ce caractère dans un article des Annales (Rey, 1973), et les cartes les plus récentes de hiérarchie urbaine confirment la stabilité de cette option (fig. 8). Les centres s'emboîtent régulièrement selon leur niveau hiérarchique et l'on souhaiterait faire quelques mesures pour vérifier la régularité de la configuration. Espacés sur l'ensemble du pays, les centres de premier ordre assurent l'armature urbaine du territoire. Les chefs-lieux de préfecture en constituent l'échelon suivant, et leur sélection comme chef-lieu en 1967 a parfois retenu la position géographique centrale dans l'unité administrative avant le poids démographique (exemple de Slobozia au lieu de Calarasi dans le département de Ialomita). La constitution de cette trame hiérarchique des centres est indissociable enfin de l'effort mené au niveau inférieur des bourgades rurales. La systématisation de l'habitat rural est en cours de réalisation, avec pour objectif de réduire le nombre de villages au profit des bourgs au centre d'unités communales agrandies. Cet aménagement est certainement un champ d'observation privilégié du mécanisme d'équilibrage territorial et du processus de transformation sociale par le biais de l'urbanisation.

Que conclure de ces premières observations sur les réalisations en matière d'équilibre du réseau urbain? Elles laissent la tentation d'identifier là une application du modèle de Christaller, fondé sur 


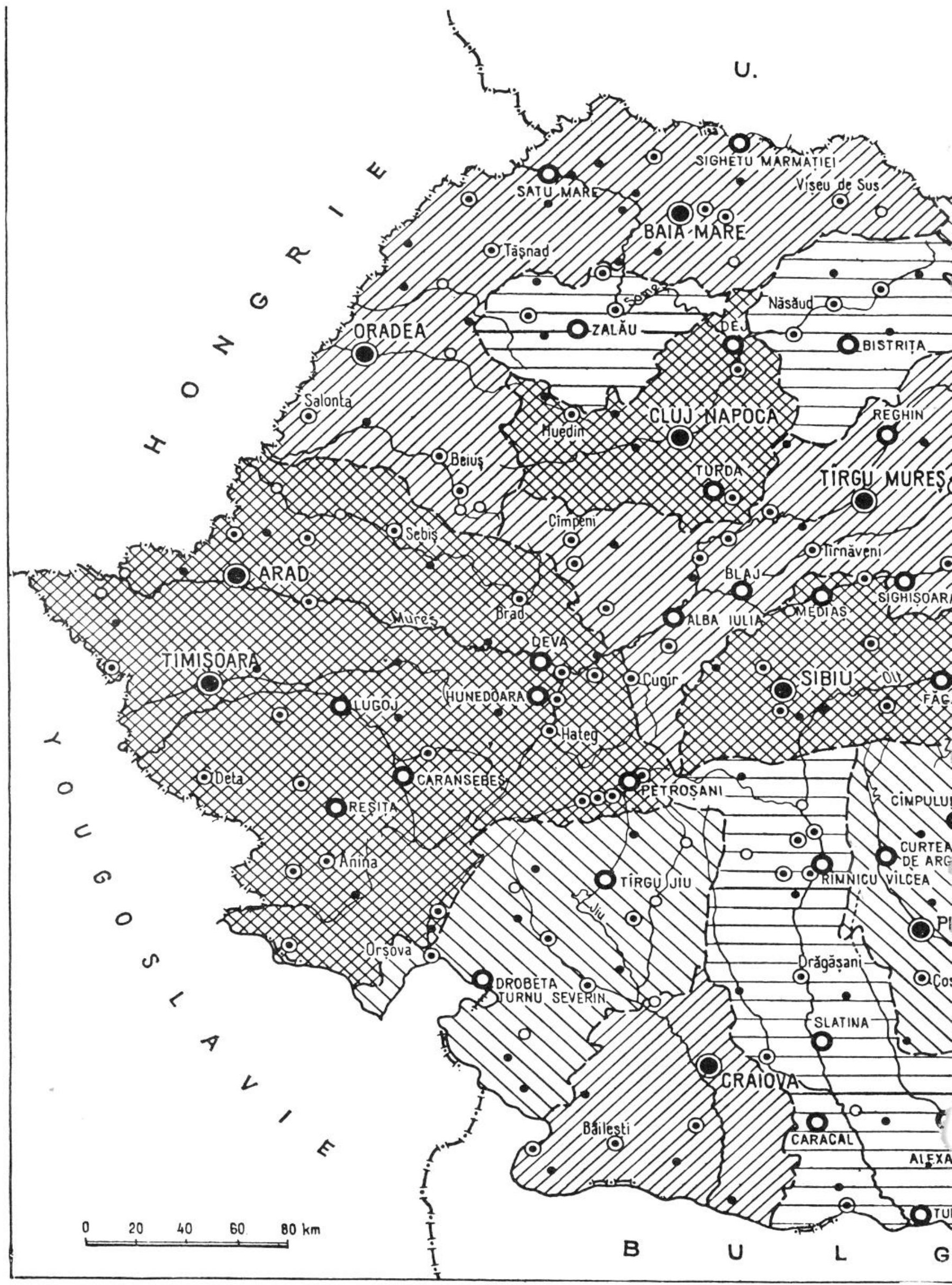

Fig. 8 - NIVEAUX DE HIÉRARCHIE DES VILLES d'après V. Cucu et al. (1980). 


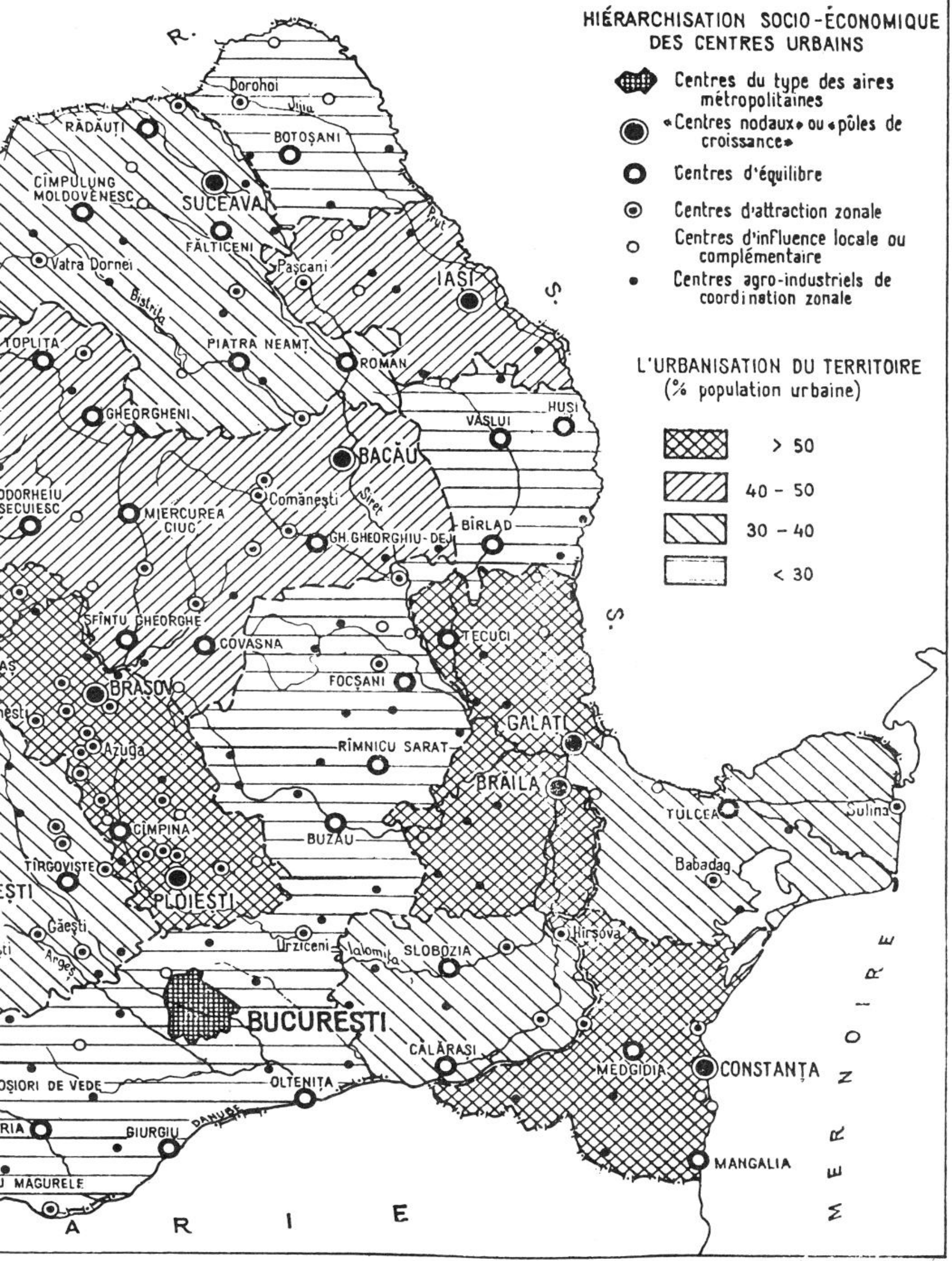


l'équi-répartition spatiale des centres de même niveau. Observée et conçue dans une économie capitaliste, cette configuration de trame paraissait à Christaller traduire de la manière optimale le principe du marché. Réalisée en économie socialiste, il y a substitution de la cause mais conservation de la forme territoriale: l'objectif d'égalité de desserte des "avantages » de la ville à l'ensemble de la population a remplacé celui du marché. Or ce souci majeur «d'équilibre dans la croissance urbaine» soulève certaines questions. Par quels moyens se réalise concrètement la croissance régulée? Quelles réelles modifications des conditions du mode de vie sous-tendent la notion de progrès d'urbanisation? En quoi un tel objectif est-il peut-être plus adapté à une période de difficultés économiques générales que celui de la croissance des grands centres urbains? Et surtout, - question de fond devant laquelle achoppe tout aménagement du territoire — jusqu'où le choix politique d'une équi-répartition des forces urbaines est-il compatible avec les économies d'échelle dont la grande ville serait porteuse? Rappelons que l'amélioration générale des niveaux de vie a été retenue comme prioritaire; or elle passe par des gains de productivité, dont on sait qu'ils sont en partie produits par ces économies d'échelle.

De l'approche de la croissance urbaine, que j'ai appréhendée dans sa dimension quantitative, je retiendrai les conclusions suivantes. La forte ruralité et la vitalité démographique dans laquelle s'inscrit cette croissance sont des marques spécifiquement roumaines. La volonté de réaliser une hiérarchie territoriale équilibrée et de contrebalancer le caractère dissymétrique du développement industriel par une diffusion des services est plutôt une spécificité de l'appartenance au système socialiste. Quant à l'homogénéisation des profils d'activités des villes qui accompagne leur développement et à la place croissante du secteur tertiaire dans leur dynamique, ce sont deux composantes beaucoup plus banales de l'évolution des systèmes urbains des pays à économie développée, en régime capitaliste ou socialiste.

Beaucoup d'aspects de la croissance urbaine roumaine n'ont pas été abordés ici. Or certains ont déjà fait l'objet de nombreux travaux; en particulier l'évolution de la morphologie intra-urbaine, l'évolution des aires d'attraction de main-d'œuvre et la desserte en transports, l'évolution d'une grande ville et/ou d'un réseau urbain régional, la mesure de certaines modifications introduites sur l'environnement par l'extension urbaine (en particulier des études de climatologie urbaine). D'autres aspects sont encore peu traités, alors qu'ils prennent une place croissante dans la littérature occidentale sur la ville; ainsi en est-il de l'étude du financement et des coûts de la croissance urbaine, de l'étude des "acteurs" qui, 
concrètement, participent aux politiques locales d'affectations territoriales (en zones à bâtir, zones de réserves territoriales, zones agricoles...); de l'étude de la nouvelle société urbaine dans sa dimension culturelle autant que sociologique...

\section{Bibliographie}

J'ai limité cette bibliographie aux articles et ouvrages roumains récents les plus directement liés au thème de la croissance urbaine nationale et cités dans cette communication. Pour des analyses antérieures relatives à la géographie urbaine roumaine, je renvoie à Rey V.:

1. Chronique bibliographique, Annales de Géographie (1972, pp. 711-729).

2. "Brasov, une vocation urbaine" (1975), Bibliothèque Nationale.

3. "La Roumanie essai d'analyse régionale" (1975), Paris, SEDES.

(RRGGG: Revue Roumaine de Géologie, Géophysique, Géographie).

Apavaloaei M., Lupu Bratiloveanu N., (1980), "Les établissements industriels de RSR et leur influence sur la mobilité de la population", RRGGG, pp. 155-160.

Constantinescu M., Stahl H.H., Dragan I., (1974), Urban growth processes in Romania, Meridiane Publishing House, Bucarest, $280 \mathrm{p}$.

Cucu V. (1970), Orasele Romaniei, Editura Stiintifica, Bucarest, 152 p.

Cucu V., Deica P., (1978), "Le processus d'urbanisation en Roumanie", RRGGG, pp. 239-250.

Ianos I., Olaru M. (1980), "Contributions to the study of daily commutation in CarasSeverin county ", RRGGG, pp. 161-166.

Measnicov I., Hristache I., Trebici V., (1977), Demografia oraselor Romaniei, Editura stiintifica, Bucarest, 223 p.

Panaite L., Chitu M., Caloianu N. (1978), "First rank polarizing centres in Romania's urban settlement network», RRGGG, pp. 251-256.

Sandru I., Cucu V., Poghirc P., (1963), "Contribution géographique à la classification des villes de la République populaire roumaine", Annales de Géographie, Paris, pp. 162-180.

Stefanescu 1. (1980), "Demographic types in Romania and their geographical distribution", RRGGG, pp. 133-138.

Tufescu V. (1980), "Sur l'urbanisation des villages de Roumanie " RRGGG, pp. 139146.

Résumé. - A quelles tendances démographiques, économiques et spatiales se rattache l'urbanisation roumaine? Elle atteint 10,3 millions de citadins $148 \%$ de la population totale) et a presque doublé l'effectif des villes. L'auteur inscrit les modalités de cette urbanisation dans le cadre idéologique du socialisme d'Etat et dans les cadres plus généraux relatifs aux modèles de croissance urbaine.

La forte ruralité et la vitalité démographique ont d'abord nourri, de manière spécifique, la croissance, laquelle s'est appuyée sur des mouvements migratoires dont la forme et l'ampleur restent dans la dépendance des modeles de gravité. Toutefois, ce mode d'urbanisation paraît être en fin de phase, tandis que se développent une mobilité temporaire et la suburbanistion. Parallèlement, 
le secteur tertiaire semble prendre le relais sur le secteur secondaire, ce qui soulève l'importante question de la nature motrice du tertiaire socialiste.

Territorialement, il y a eu une réduction des écarts entre les niveaux d'urbanisation, mais avec un mode de recrutement urbain et des conséquences sur les campagnes profondément différents de part et d'autre d'une diagonale N.O.-S.E. Cinq cartes des nouvelles unités urbaines apparues depuis le début du siècle montrent les phases de densification du réseau national (réseau régulier, réseau spécifique). La transformation spatiale volontaire du semis de peuplement paraît, à travers cette étude, plus aisée que l'infléchissement de la hiérarchie des tailles, puisque les deux graphiques "rang-taille" de 1930 et 1977 sont similaires.

Abstract. - What are the demographic, economic and spatial trends of Romanian urbanization? Citydwellers are now as much as 10.3 millions $(48 \%$ of total population) and city population has nearly doubled. The author describes the characteristics of this urbanization according to the State-ruled socialist ideological models and also to the more general usual urban-growth models.

First fed by the large rural population and the important natural growth, city growth is the result of migrations whose mode and size show gravity-like patterns. However this kind of urbanization seems to be phasing out right now, while temporary migrations and suburbanization develop altogether. Besides, the tertiary sector seems to outnumber the industry sector, which raises the crucial problem of the driving role of the tertiary sector within a socialist economy.

Spatial differences between the urbanization levels have attenuated but the origin of city newcomers and the consequences of rural exodus on the countryside notably differ on each side of a NW-SE diagonal. Five maps of the new conurbations that have developed since the beginning of this century show the densification steps of the national urban network (regular network, specific network). The voluntary spatial transformation of the population densities seems, according to this study, easier than the reorientation of city-size order, since the two rank-size graphs of 1930 and 1977 are alike. 